\title{
Derivation and Extensions of the Linear Feedback Particle Filter based on Duality Formalisms
}

\author{
Jin-Won Kim, Amirhossein Taghvaei and Prashant G. Mehta
}

\begin{abstract}
This paper is concerned with a duality-based approach to derive the linear feedback particle filter (FPF). The FPF is a controlled interacting particle system where the control law is designed to provide an exact solution for the nonlinear filtering problem. For the linear Gaussian special case, certain simplifications arise whereby the linear FPF is identical to the square-root form of the ensemble Kalman filter. For this and for the more general nonlinear non-Gaussian case, it has been an open problem to derive/interpret the FPF control law as a solution of an optimal control problem. In this paper, certain duality-based arguments are employed to transform the filtering problem into an optimal control problem. Its solution is shown to yield the deterministic form of the linear FPF. An extension is described to incorporate stochastic effects due to noise leading to a novel homotopy of exact ensemble Kalman filters. All the derivations are based on duality formalisms.
\end{abstract}

\section{INTRODUCTION}

Amongst the many derivations of the Kalman filter, a particularly appealing one (to control theorists) is based on duality arguments; cf., [1, Chapter 7]. In this derivation, the linear Gaussian filtering (estimation) problem is modeled as an optimization problem involving minimization of the mean-squared error (variance) of the estimate. By designing a suitable dual process (evolving in backward time), the optimization problem is transformed into a finite-horizon deterministic linear quadratic (LQ) optimal control problem. The solution to this LQ problem yields the Kalman filter.

The duality theorem originally appeared in Kalman's celebrated paper [2]. The duality relationship between optimal control and linear filtering has proved to be very useful: (i) it provides an interpretation of the Kalman filter as the minimum variance estimator; (ii) it helps explain why the filtering equation for the error covariance is same as the dynamic Ricatti equation (DRE) of the optimal control; and (iii) quantitative results on the asymptotic properties of the solution of the DRE have been used to derive results on asymptotic stability of the linear filter [3].

Given the historical significance of these problems, several extensions have been considered. Early on, the linear duality was related to the duality in nonlinear programming [4]. Extensions of the duality principle to constrained linear estimation are described in [5].

Extensions of the duality theory to nonlinear non-Gaussian settings is a closely related subject of historical importance

Financial support from the NSF CMMI grant 1462773 is gratefully acknowledged.

J-W. Kim, A. Taghvaei and P. G. Mehta are with the Coordinated Science Laboratory and the Department of Mechanical Science and Engineering at the University of Illinois at Urbana-Champaign (UIUC); Corresponding email: mehtapg@illinois.edu beginning with Mortensen's maximum-likelihood estimator [6]. Over the years, there have been a number of important contributions in this area, e.g., the use of logarithmic (Hopf-Cole) transformation to convert the filtering equation into the Hamilton-Jacobi-Bellman (HJB) equation of optimal control [7]. An information theoretic interpretation for the same appears in [8]. More recently, these considerations have led to the development and application of the path integral approaches to filtering and smoothing problems [9]. For a recent review with a comprehensive reference list, cf., [10].

The goal of this paper is to generalize and apply Kalman's duality principle for the purposes of deriving particle filters, in the tractable linear Gaussian settings. Specifically, the linear feedback particle filter (also referred to as the squareroot form of the ensemble Kalman filter) is derived by extending results from the classical duality theory.

As some pertinent background, the feedback particle filter (FPF) is an example of a controlled interacting particle system to approximate the solution of the continuous-time nonlinear filtering problem. In FPF, the importance sampling step of the conventional particle filter is replaced with feedback control. Other steps such as resampling, reproduction, death or birth of particles are altogether avoided. A salient feature of the FPF is that it is an exact filter even in nonlinear-non-Gaussian settings. An expository review of the continuous-time filters including the progression from the Kalman filter (1960s) to the ensemble Kalman filter (1990s) to the feedback particle filter (2010s) appears in [11].

The contributions of this paper are as follows: This paper is the first to present a derivation of linear FPF/ensemble Kalman filters based on duality considerations. The adaptation of the duality formalisms to the particle filter, as proposed here, is also original. These considerations are used to obtain a novel homotopy of stochastic and deterministic (linear) particle filters all of which are exact.

Although it is not an explicit focus of this paper, the derivation potentially provides new tools to analyze and interpret the optimality properties of the filter, in terms of the simulation variance of its estimates. These properties are important in applications of ensemble Kalman filters, in particular, for high-dimension problems.

The outline of the remainder of this paper is as follows: Sec. II provides the background on FPF. In Sec. III, the duality formalism for the particle filter is introduced to construct an optimization problem. The solution to this problem leading to the deterministic form of the linear FPF is presented in Sec. IV. The generalization to the stochastic case appears on Sec. V. All the proofs appear in the Appendix. 


\section{PRELIMINARIES AND BACKGROUND}

In this paper, we consider the linear Gaussian filtering problem:

$$
\begin{aligned}
& \mathrm{d} X_{t}=A_{t} X_{t} \mathrm{~d} t+\mathrm{d} B_{t} \\
& \mathrm{~d} Z_{t}=C_{t} X_{t} \mathrm{~d} t+\mathrm{d} W_{t}
\end{aligned}
$$

where $X_{t} \in \mathbb{R}^{d}$ is the (hidden) state at time $t, Z_{t} \in \mathbb{R}^{m}$ is the observation; $A_{t}, C_{t}$ are matrices of appropriate dimension whose elements are continuous in $t$; and $\left\{B_{t}\right\},\left\{W_{t}\right\}$ are mutually independent Wiener processes taking values in $\mathbb{R}^{d}$ and $\mathbb{R}^{m}$, respectively. The covariance associated with $\left\{B_{t}\right\}$ and $\left\{W_{t}\right\}$ are denoted by $Q_{t}$ and $R_{t}$, respectively. The initial condition $X_{0}$ is drawn from a Gaussian distribution $\mathcal{N}\left(m_{0}, \Sigma_{0}\right)$, independent of $\left\{\boldsymbol{B}_{t}\right\}$ or $\left\{W_{t}\right\}$. It is assumed that the covariance matrices $Q_{t}, R_{t}$ and $\Sigma_{0}$ are strictly positive definite for all $t$. The filtering problem is to compute the posterior distribution $\mathrm{P}\left(X_{t} \mid \mathcal{Z}_{t}\right)$ where $\mathcal{Z}_{t}:=\sigma\left(Z_{s} ; s \in[0, t]\right)$ denotes the filtration (the time-history of observations).

For the linear Gaussian problem (1a)-(1b), the posterior distribution $\mathrm{P}\left(X_{t} \mid \mathcal{Z}_{t}\right)$ is Gaussian $\mathcal{N}\left(m_{t}, \Sigma_{t}\right)$, whose mean and covariance are given by the Kalman-Bucy filter [12]:

$$
\begin{aligned}
& \mathrm{d} m_{t}=A_{t} m_{t} \mathrm{~d} t+\mathrm{K}_{t}\left(\mathrm{~d} Z_{t}-C_{t} m_{t} \mathrm{~d} t\right) \\
& \frac{\mathrm{d} \Sigma_{t}}{\mathrm{~d} t}=A_{t} \Sigma_{t}+\Sigma_{t} A_{t}^{\top}+Q_{t}-\Sigma_{t} C^{\top} R_{t}^{-1} C \Sigma_{t}
\end{aligned}
$$

where $\mathrm{K}_{t}:=\Sigma_{t} C^{\top} R_{t}^{-1}$ is the Kalman gain and the filter is initialized with the Gaussian prior $\left(m_{0}, \Sigma_{0}\right)$.

Feedback particle filter (FPF) is a controlled interacting particle system to approximate the Kalman filter ${ }^{1}$. In the following, the McKean-Vlasov stochastic differential equation (sde) are presented for the linear FPF algorithm. For these models, the state at time $t$ is denoted as $\bar{X}_{t}$. Two types of FPF algorithm have been described in the literature [14], [15]:

(A) Stochastic linear FPF: The state $\bar{X}_{t}$ evolves according to the McKean-Vlasov sde:

$$
\mathrm{d} \bar{X}_{t}=A_{t} \bar{X}_{t} \mathrm{~d} t+\mathrm{d} \bar{B}_{t}+\overline{\mathrm{K}}_{t}\left(\mathrm{~d} Z_{t}-\frac{C_{t} \bar{X}_{t}+C_{t} \bar{m}_{t}}{2} \mathrm{~d} t\right)
$$

where $\overline{\mathrm{K}}_{t}=\bar{\Sigma}_{t} C_{t}^{\top} R_{t}^{-1}$ is the Kalman gain; the mean-field terms are the mean $\bar{m}_{t}=\mathrm{E}\left[\bar{X}_{t} \mid \mathcal{Z}_{t}\right]$ and the covariance $\bar{\Sigma}_{t}=$ $\mathrm{E}\left[\left(\bar{X}_{t}-\bar{m}_{t}\right)\left(\bar{X}_{t}-\bar{m}_{t}\right)^{\top} \mid \mathcal{Z}_{t}\right] ;\left\{\bar{B}_{t}\right\}$ is an independent copy of the process noise $\left\{B_{t}\right\}$; and the initial condition $\bar{X}_{0} \sim \mathcal{N}\left(m_{0}, \Sigma_{0}\right)$.

(B) Deterministic linear FPF: The McKean-Vlasov sde is:

$$
\mathrm{d} \bar{X}_{t}=A_{t} \bar{X}_{t} \mathrm{~d} t+\frac{1}{2} Q_{t} \bar{\Sigma}_{t}^{-1}\left(\bar{X}_{t}-\bar{m}_{t}\right) \mathrm{d} t+\overline{\mathrm{K}}_{t}\left(\mathrm{~d} Z_{t}-\frac{C_{t} \bar{X}_{t}+C_{t} \bar{m}_{t}}{2} \mathrm{~d} t\right)
$$

where (as before) $\overline{\mathrm{K}}_{t}=\bar{\Sigma}_{t} C_{t}^{\top} R_{t}^{-1}$ is the Kalman gain; the mean $\bar{m}_{t}=\mathrm{E}\left[\bar{X}_{t} \mid \mathcal{Z}_{t}\right]$ and the covariance $\bar{\Sigma}_{t}=\mathrm{E}\left[\left(\bar{X}_{t}-\bar{m}_{t}\right)\left(\bar{X}_{t}-\right.\right.$ $\left.\left.\bar{m}_{t}\right)^{\top} \mid \mathcal{Z}_{t}\right]$; the initial condition $\bar{X}_{0} \sim \mathcal{N}\left(m_{0}, \Sigma_{0}\right)$.

The difference between the two algorithms is that the process noise term $\mathrm{d} \bar{B}_{t}$ in the stochastic FPF (3) is replaced by $\frac{1}{2} Q_{t} \bar{\Sigma}_{t}^{-1}\left(\bar{X}_{t}-\bar{m}_{t}\right)$ in the deterministic FPF (4).

\footnotetext{
${ }^{1}$ Although the considerations of this paper are limited to the linear Gaussian problem (1a)-(1b), the FPF algorithm is more broadly applicable to nonlinear non-Gaussian filtering problems [13], [14].
}

The following Proposition, borrowed from [15], shows that both the filters are exact:

Proposition 1: (Theorem 1 in [15]) Consider the linear Gaussian filtering problem (1a)-(1b), and the linear FPF (Eq. (3) or Eq. (4)). If $\mathrm{P}\left(X_{0}\right)=\mathrm{P}\left(\bar{X}_{0}\right)$ then

$$
\mathrm{P}\left(\bar{X}_{t} \mid \mathcal{Z}_{t}\right)=\mathrm{P}\left(X_{t} \mid \mathcal{Z}_{t}\right), \quad \forall t>0
$$

Therefore, $m_{t}=\bar{m}_{t}$ and $\Sigma_{t}=\bar{\Sigma}_{t}$.

In a numerical implementation, the filter is simulated with $N$ interacting particles where $N$ is typically large. The filter state is $\left\{X_{t}^{i}: 1 \leq i \leq N\right\}$, where $X_{t}^{i}$ is the state of the $i^{\text {th }}$-particle at time $t$. The evolution of $X_{t}^{i}$ is obtained upon empirically approximating the mean-field terms. For example, the finite$N$ algorithm for the stochastic FPF is as follows:

(A) Finite- $N$ stochastic FPF: The evolution of $X_{t}^{i}$ is given by the sde:

$$
\mathrm{d} X_{t}^{i}=A_{t} X_{t}^{i} \mathrm{~d} t+\mathrm{d} B_{t}^{i}+\mathrm{K}_{t}^{(N)}\left(\mathrm{d} Z_{t}-\frac{C_{t} X_{t}^{i}+C_{t} m_{t}^{(N)}}{2} \mathrm{~d} t\right)
$$

where $\mathrm{K}_{t}^{(N)}:=\Sigma_{t}^{(N)} C_{t}^{\top} R_{t}^{-1} ;\left\{B_{t}^{i}\right\}_{i=1}^{N}$ are independent copies of $B_{t} ; X_{0}^{i} \stackrel{\text { i.i.d }}{\sim} \mathcal{N}\left(m_{0}, \Sigma_{0}\right)$ for $i=1,2, \ldots, N$; and the empirical approximations of the two mean-field terms are as follows:

$$
m_{t}^{(N)}:=\frac{1}{N} \sum_{j=1}^{N} X_{t}^{i}, \Sigma_{t}^{(N)}:=\frac{1}{N-1} \sum_{j=1}^{N}\left(X_{t}^{i}-m_{t}^{(N)}\right)\left(X_{t}^{i}-m_{t}^{(N)}\right)^{\top}
$$

The McKean-Vlasov sde (3) represents the mean-field limit (as $N \rightarrow \infty$ ) of the finite- $N$ system (5).

\section{DUALITY}

\section{A. Classical duality}

Classical duality is concerned with the problem of constructing a minimum variance estimator for the random variable $a^{\top} X_{T}$, where $X_{T}$ is the hidden state (defined according to the model (1a)) at (some fixed) time $T>0$ and $a \in \mathbb{R}^{d}$ is an arbitrary (but known) vector.

Given observations $Z_{t}$ for $t \in[0, T]$ (defined according to the model (1b)), the assumed linear structure for the causal estimator is as follows:

$$
S_{T}=b_{T}^{\top} m_{0}+\int_{0}^{T} u_{t}^{\top} \mathrm{d} Z_{t}
$$

The filter is thus parametrized by the vector $b_{T} \in \mathbb{R}^{d}$ and the (possibly) time-dependent control input $u:[0, T] \rightarrow \mathbb{R}^{m}$. The latter is denoted as $u_{t}$.

The optimal filter parameters are obtained by solving the following mean-squared optimization problem:

$$
\min _{b_{T}, u_{t}} \mathrm{E}\left[\left|S_{T}-a^{\top} X_{T}\right|^{2}\right]
$$

subject to the following constraints: (1a)-(1b) for the processes $X_{t}$ and $Z_{t}$, and (6) for the random variable $S_{T}$.

In the duality based derivation of Kalman filter, the optimization problem is converted into a deterministic optimal control problem by introducing a suitable dual process; cf., [1, Chapter 7]. The solution of the optimal control problem yields the optimal control input $u_{t}$ and vector $b_{T}$. By expressing the estimator (6) in its differential form, one obtains the Kalman filter. 


\section{B. Duality for the particle filter}

A particle filter empirically approximates the posterior distribution. By the $L^{2}$ optimality property of the posterior, the particle filter can be used to obtain a mean-squared estimate of any arbitrary function of the hidden state.

The particle analogue of (6) is as follows: Define $N$ random variables $\left\{S_{T}^{i}: 1 \leq i \leq N\right\}$ according to

$$
S_{T}^{i}=\sum_{j=1}^{N}\left(b_{T}^{i j}\right)^{\top} X_{0}^{j}+\int_{0}^{T}\left(u_{t}^{i}\right)^{\top} \mathrm{d} Z_{t}
$$

where the filter parameters now are $b_{T}^{i j} \in \mathbb{R}^{d}$ for $i, j=1, \ldots, N$ and the control input $u^{i}:[0, T] \rightarrow \mathbb{R}^{m}$ for $i=1, \ldots, N$. As before, the filter state depends linearly on the known data observations up to time $T$ and the initial condition $X_{0}^{j}$ for $j=1, \ldots, N$. The initial condition $X_{0}^{j} \stackrel{\text { i.i.d }}{\sim} \mathcal{N}\left(m_{0}, \Sigma_{0}\right)$. Such a sampling ensures that the filter provides consistent estimates (as $N \rightarrow \infty$ ) at time $T=0$.

The optimal filter parameters are chosen by solving the following mean-squared optimization problem:

$$
\min _{b_{T}^{i j}, u_{t}^{i}} \mathrm{E}\left[\left|\frac{1}{N} \sum_{i=1}^{N} f\left(S_{T}^{i}\right)-f\left(a^{\top} X_{T}\right)\right|^{2}\right]
$$

subject to the constraints: (1a)-(1b) for the processes $X_{t}$ and $Z_{t}$, and (7) for the random variables $S_{T}^{i}$. This is a multi-objective optimization problem (as the function $f$ is arbitrary). With the choice of $f(x)=x$, one obtains the optimization problem considered in classical duality.

There is a modeling trade-off here: Ideally, one would want to consider a large enough class of functions $f$, and the filter parameters $b$ and $u$, that can represent and approximate the posterior distribution. However, the class may not be too large because one would still want to be able to solve the multi-objective optimization problem.

In order to make the analysis tractable, the following simplifications are proposed:

Step 1: Consider the following simplifications for the filter parameters:

$$
\begin{aligned}
u_{t}^{i} & :=u_{t} \\
\sum_{j=1}^{N}\left(b_{T}^{i j}\right)^{\top} X_{0}^{j} & :=c_{T}^{\top}\left(X_{0}^{i}-X_{0}^{(N)}\right)+b_{T}^{\top} X_{0}^{(N)}
\end{aligned}
$$

where $X_{0}^{(N)}:=\frac{1}{N} \sum_{i=1}^{N} X_{0}^{i}$ is the empirical mean. Using this new parametrization, the filter (7) becomes

$$
S_{T}^{i}=c_{T}^{\top}\left(X_{0}^{i}-X_{0}^{(N)}\right)+b_{T}^{\top} X_{0}^{(N)}+\int_{0}^{T} u_{t}^{\top} \mathrm{d} Z_{t}
$$

Step 2: The mean-field limit of the estimator is obtained by letting $N \rightarrow \infty$. In the limit, the random variable is denoted as $\bar{S}_{T}$. The mean-field counterpart of the filter (8) is

$$
\bar{S}_{T}=c_{T}^{\top}\left(\bar{X}_{0}-m_{0}\right)+b_{T}^{\top} m_{0}+\int_{0}^{T} u_{t}^{\top} \mathrm{d} Z_{t}
$$

The optimization problem is to chose the filter parameters $b_{T}, c_{T}$ and $u_{t}$ to minimize the following mean-squared cost

$$
\min _{b_{T}, c_{T}, u_{t}} \mathrm{E}\left[\left|\mathrm{E}\left[f\left(\bar{S}_{T}\right) \mid \mathcal{Z}_{T}\right]-f\left(a^{\top} X_{T}\right)\right|^{2}\right]
$$

Step 3: In the final step, we restrict the class of functions to $f(x)=x$ and $f(x)=x^{2}$. The justification for considering only linear and quadratic functions is that the density of the random variable $\bar{S}_{T}$ is Gaussian (see also the Remark 1 at the end of this section).

\section{Optimization problem}

In summary, the mathematical problem is a bi-objective optimization problem:

$$
\min _{b_{T}, c_{T}, u_{t}}\left(\mathrm{E}\left[\left|\mathrm{E}\left[\bar{S}_{T} \mid \mathcal{Z}_{T}\right]-a^{\top} X_{T}\right|^{2}\right], \mathrm{E}\left[\left|\mathrm{E}\left[\bar{S}_{T}^{2} \mid \mathcal{Z}_{T}\right]-\left(a^{\top} X_{T}\right)^{2}\right|^{2}\right]\right)
$$

subject to the respective constraints for the state, observation, and the estimator:

$$
\begin{aligned}
\mathrm{d} X_{t} & =A_{t} X_{t} \mathrm{~d} t+\mathrm{d} B_{t}, \quad X_{0} \sim \mathcal{N}\left(m_{0}, \Sigma_{0}\right) \\
\mathrm{d} Z_{t} & =C_{t} X_{t} \mathrm{~d} t+\mathrm{d} W_{t} \\
\bar{S}_{T} & =b_{T}^{\top} m_{0}+c_{T}^{\top}\left(\bar{X}_{0}-m_{0}\right)+\int_{0}^{T} u_{t}^{\top} \mathrm{d} Z_{t}, \quad \bar{X}_{0} \sim \mathcal{N}\left(m_{0}, \Sigma_{0}\right)
\end{aligned}
$$

where the noise processes $W_{t}$ and $B_{t}$, and the initial conditions $X_{0}$ and $\bar{X}_{0}$, are all assumed to be mutually independent; recall that $\mathcal{Z}_{t}:=\sigma\left(Z_{s} ; s \in[0, t]\right)$ denotes the time-history of observations up to time $t$ (filtration).

A solution to this problem appears in the following section.

\section{DETERMINISTIC PARTICLE FILTER VIA DUALITY}

Denote $\Sigma_{t}$ to be the solution of the DRE (2b) with the initial condition $\Sigma_{0}$, and $\mathrm{K}_{t}:=\Sigma_{t} C^{\top} R_{t}^{-1}$ is the Kalman gain.

Define the following state transition matrices:

$$
\begin{gathered}
\frac{\mathrm{d} \Phi}{\mathrm{d} t}(t ; \tau)=\left(-A_{t}^{\top}+C_{t}^{\top} \mathrm{K}_{t}^{\top}\right) \Phi(t ; \tau) \\
\frac{\mathrm{d} \Psi}{\mathrm{d} t}(t ; \tau)=\left(-A_{t}^{\top}+\frac{1}{2} C_{t}^{\top} \mathrm{K}_{t}^{\top}-\frac{1}{2} \Sigma_{t}^{-1} Q_{t}\right) \Psi(t ; \tau)
\end{gathered}
$$

with $\Phi(\tau ; \tau)=\Psi(\tau ; \tau)=I$, the identity matrix.

These definitions are useful to describe the solution to the optimization problem (10), as presented in the following Theorem. Its proof appears in the Appendix (Sec. A).

Theorem 1: Consider the optimization problem (10) subject to the constraints (11). Its solution is as follows:

$$
\begin{aligned}
b_{T} & =\Phi(0 ; T) a \\
c_{T} & =\Psi(0 ; T) a \\
u_{t} & =\mathrm{K}_{t}^{\top} \Phi(t ; T) a
\end{aligned}
$$

The solution for $c_{T}$ is unique up to a sign for the scalar $(d=1)$ case but it is not unique for the vector $(d>1)$ case.

Using the optimal parameters (14), the estimator is

$$
\begin{aligned}
\bar{S}_{T}= & a^{\top}\left(\Phi^{\top}(0 ; T) m_{0}+\Psi^{\top}(0 ; T)\left(\bar{X}_{0}-m_{0}\right)\right. \\
& \left.+\int_{0}^{T} \Phi^{\top}(t ; T) \mathrm{K}_{t} \mathrm{~d} Z_{t}\right), \quad \bar{X}_{0} \sim \mathcal{N}\left(m_{0}, \Sigma_{0}\right)
\end{aligned}
$$


This estimator is exact. That is,

$$
\mathrm{E}\left(g\left(\bar{S}_{T}\right) \mid \mathcal{Z}_{T}\right)=\mathrm{E}\left(g\left(a^{\top} X_{T}\right) \mid \mathcal{Z}_{T}\right)
$$

for all smooth test functions $g$ and vectors $a \in \mathbb{R}^{d}$.

The following Proposition provides the differential (recursive) form of the filter which also reveals the connection to the FPF algorithm (compare with formula (4) for the deterministic FPF). The proof appears in the Appendix (Sec. B).

Proposition 2: For any given $a \in \mathbb{R}^{d}$ and $T \geq 0, \bar{S}_{T}=a^{\top} \bar{X}_{T}$ where $\bar{X}_{T}$ is the strong solution of the following mean-field sde:

$$
\mathrm{d} \bar{X}_{t}=A_{t} \bar{X}_{t} \mathrm{~d} t+\mathrm{K}_{t}\left(\mathrm{~d} Z_{t}-\frac{C_{t} \bar{X}_{t}+C_{t} \bar{m}_{t}}{2} \mathrm{~d} t\right)+\frac{1}{2} Q_{t} \Sigma_{t}^{-1}\left(\bar{X}_{t}-\bar{m}_{t}\right) \mathrm{d} t
$$

where $\bar{m}_{t}=\mathrm{E}\left[\bar{X}_{t} \mid \mathcal{Z}_{t}\right]$ and the initial condition $\bar{X}_{0} \sim \mathcal{N}\left(m_{0}, \Sigma_{0}\right)$.

Remark 1: The derivation of the deterministic FPF offers an a posteriori justification of our simplifying choices for the filter parameters (in step 1) and the functions $f$ (in step 3 ). That these choices were sufficient is primarily due to the linear Gaussian nature of the problem. More generally, if the distribution is sub-Gaussian, the method of moments suggests considering $\left\{f(x)=x^{k} \mid k=1,2, \ldots\right\}$ as a class of functions [16, Section 30].

\section{Stochastic PARTICLE FILTER VIA DUALity}

In this section, we extend the deterministic filter structure (8) to now include noise terms:

$$
\begin{aligned}
S_{T}^{i}= & b_{T}^{\top} X_{0}^{(N)}+c_{T}^{\top}\left(X_{0}^{i}-X_{0}^{(N)}\right) \\
& +\int_{0}^{T} u_{t}^{\top} \mathrm{d} Z_{t}+\int_{0}^{T} v_{t}^{\top} \mathrm{d} B_{t}^{i}+\int_{0}^{T} w_{t}^{\top} \mathrm{d} W_{t}^{i}
\end{aligned}
$$

where the $\left\{B_{t}^{i}\right\}$ and $\left\{W_{t}^{i}\right\}$ are independent copies of the process noise (with covariance $Q_{t}$ ) and the measurement error (with covariance $R_{t}$ ), respectively. These are also independent of $B_{t}, W_{t}, X_{0}, X_{0}^{i}$. The design problem is to chose the filter parameters $\left\{b_{T}, c_{T}, u_{t}, v_{t}, w_{t}\right\}$.

The motivation of considering this type of filter structure is two-fold: (i) $S_{T}^{i}$ now depends linearly on all of the dataobservation, initial condition as well as the copies of the process noise and the measurement noise; and (ii) particle filters with noise terms are widely used in practice [11], [17]. For example, the stochastic FPF (3) includes noise term to simulate the effect of process noise. The ensemble Kalman filter include noise terms to simulate the effect of both process noise and measurement noise [17].

On repeating the steps 1-3, the stochastic counterpart of the optimization problem (10)-(11) is obtained as follows:

$$
\min _{\substack{b_{T}, c_{T}, u_{t}, v_{t}, w_{t}}}\left(\mathrm{E}\left[\left|\mathrm{E}\left[\bar{S}_{T} \mid \mathcal{Z}_{T}\right]-a^{\top} X_{T}\right|^{2}\right], \mathrm{E}\left[\left|\mathrm{E}\left[\bar{S}_{T}^{2} \mid \mathcal{Z}_{T}\right]-\left(a^{\top} X_{T}\right)^{2}\right|^{2}\right]\right)
$$

subject to

$$
\begin{aligned}
\mathrm{d} X_{t}= & A_{t} X_{t} \mathrm{~d} t+\mathrm{d} B_{t}, \quad X_{0} \sim \mathcal{N}\left(m_{0}, \Sigma_{0}\right) \\
\mathrm{d} Z_{t}= & C_{t} X_{t} \mathrm{~d} t+\mathrm{d} W_{t} \\
\bar{S}_{T}= & b_{T}^{\top} m_{0}+c_{T}^{\top}\left(\bar{X}_{0}-m_{0}\right)+\int_{0}^{T} u_{t}^{\top} \mathrm{d} Z_{t} \\
& +\int_{0}^{T} v_{t}^{\top} \mathrm{d} \bar{B}_{t}+\int_{0}^{T} w_{t}^{\top} \mathrm{d} \bar{W}_{t}, \quad \bar{X}_{0} \sim \mathcal{N}\left(m_{0}, \Sigma_{0}\right)
\end{aligned}
$$

A solution of the optimization problem is given in the following theorem whose proof appears in the Appendix (Sec. C). As in the deterministic case, the solution is given in terms of certain state transition matrices. The state-transition matrix $\Phi$ is as defined in (12). The state transition matrix $\Psi$ is modified to the following:

$$
\frac{\mathrm{d} \Psi}{\mathrm{d} t}(t ; \tau)=\left(-A_{t}^{\top}+\frac{1+\gamma_{2}^{2}}{2} C_{t}^{\top} \mathrm{K}_{t}^{\top}-\frac{1-\gamma_{1}^{2}}{2} \Sigma_{t}^{-1} Q_{t}\right) \Psi(t ; \tau)
$$

where $\gamma_{1}$ and $\gamma_{2}$ are real-valued parameters. With $\gamma_{1}=\gamma_{2}=0$, one obtains the original definition (13) for $\Psi$.

Theorem 2: Consider the optimization problem (16) subject to the dynamic constraints (17). One solution of the optimization problem is as follows:

$$
\begin{aligned}
b_{T} & =\Phi(0 ; T) a \\
c_{T} & =\Psi(0 ; T) a \\
u_{t} & =\mathrm{K}_{t}^{\top} \Phi(t ; T) a \\
v_{t} & =\gamma_{1} \Psi(t ; T) a \\
w_{t} & =\gamma_{2} \mathrm{~K}_{t}^{\top} \Psi(t ; T) a
\end{aligned}
$$

where the values of the parameters $\gamma_{1}$ and $\gamma_{2}$ can be arbitrarily chosen. The resulting optimal estimator is:

$$
\begin{aligned}
\bar{S}_{T}= & a^{\top}\left(\Phi^{\top}(0 ; T) m_{0}+\Psi^{\top}(0 ; T)\left(\bar{X}_{0}-m_{0}\right)\right. \\
& +\int_{0}^{T} \Phi^{\top}(t ; T) \mathrm{K}_{t} \mathrm{~d} Z_{t}+\gamma_{1} \int_{0}^{T} \Psi^{\top}(t ; T) \mathrm{d} \bar{B}_{t} \\
& \left.+\gamma_{2} \int_{0}^{T} \Psi^{\top}(t ; T) \mathrm{K}_{t} \mathrm{~d} \bar{W}_{t}\right)
\end{aligned}
$$

The estimator is exact for every choice of the parameter values $\gamma_{1}$ and $\gamma_{2}$. That is,

$$
\mathrm{E}\left(g\left(\bar{S}_{T}\right) \mid \mathcal{Z}_{T}\right)=\mathrm{E}\left(g\left(a^{\top} X_{T}\right) \mid \mathcal{Z}_{T}\right)
$$

for all smooth test functions $g$ and vectors $a \in \mathbb{R}^{d}$.

The differential form of the filter is given in the following Proposition whose proof appears in the Appendix.

Proposition 3: For any given $a \in \mathbb{R}^{n}$ and $T \geq 0, \bar{S}_{T}=a^{\top} \bar{X}_{T}$ where $\bar{X}_{T}$ is the strong solution of the following mean-field sde:

$$
\begin{aligned}
\mathrm{d} \bar{X}_{t}= & A_{t} \bar{X}_{t} \mathrm{~d} t+\gamma_{1} \mathrm{~d} \bar{B}_{t}+\frac{1-\gamma_{1}^{2}}{2} Q_{t} \Sigma_{t}^{-1}\left(\bar{X}_{t}-\bar{m}_{t}\right) \mathrm{d} t \\
& +\mathrm{K}_{t}\left(\mathrm{~d} Z_{t}-C_{t}\left(\frac{\left(1+\gamma_{2}^{2}\right) \bar{X}_{t}+\left(1-\gamma_{2}^{2}\right) \bar{m}_{t}}{2}\right) \mathrm{d} t+\gamma_{2} \mathrm{~d} \bar{W}_{t}\right)
\end{aligned}
$$

where $\bar{m}_{t}=\mathrm{E}\left[\bar{X}_{t} \mid \mathcal{Z}_{t}\right]$ and the initial condition $\bar{X}_{0} \sim \mathcal{N}\left(m_{0}, \Sigma_{0}\right)$. 
Remark 2: The parameters $\gamma_{1}$ and $\gamma_{2}$ parametrize a homotopy of ensemble Kalman filters/linear FPFs, all of which are exact in the linear Gaussian settings.

1) For $\gamma_{1}=\gamma_{2}=0$, one obtains the deterministic form of the linear FPF (Eq. (4)).

2) For $\gamma_{1}=1$ and $\gamma_{2}=0$, one obtains the stochastic linear FPF (Eq. (3)).

3) For $\gamma_{1}=\gamma_{2}=1$, one obtains the original form of the ensemble Kalman filter [11, Eq. (7)] where a copy of measurement noise is introduced in the error.

By varying the parameters $\gamma_{1}$ and $\gamma_{2}$ in the range $[0,1]$, one goes from the two stochastic filters to the deterministic filter. The filters are exact for arbitrary (even time-varying) values of parameters $\gamma_{1}$ and $\gamma_{2}$.

\section{CONCLUSION AND DIRECTIONS FOR FUTURE WORK}

In this paper, a novel homotopy of exact linear Gaussian particle filters is derived, based on a certain extension of the classical duality. The filter is also related to the linear FPF and the ensemble Kalman filter.

There are several possible directions of future work. The first direction is to extend the proposed duality framework to now incorporate the finite- $N$ effects. Although it was not an explicit focus of this paper, the relationship to optimal control potentially provides new tools to analyze and interpret the optimality properties of the particle filter, in terms of the simulation variance of its estimates. The other direction is to extend the duality framework to nonlinear and non-Gaussian settings. Note that FPF algorithm is known to be exact in these settings. If possible, it will certainly be of interest to derive the general FPF using the duality framework.

\section{REFERENCES}

[1] K. J. Åström, Introduction to Stochastic Control Theory. Academic Press, 1970.

[2] R. E. Kalman, "A new approach to linear filtering and prediction problems," Journal of basic Engineering, vol. 82, no. 1, pp. 35-45, 1960.

[3] D. Ocone and E. Pardoux, "Asymptotic stability of the optimal filter with respect to its initial condition," SIAM Journal on Control and Optimization, vol. 34, no. 1, pp. 226-243, 1996.

[4] K. W. Simon and A. R. Stubberud, "Duality of linear estimation and control," Journal of Optimization Theory and Applications, vol. 6, no. 1 , pp. 55-67, 1970.

[5] G. C. Goodwin, J. A. de Doná, M. M. Seron, and X. W. Zhuo, "Lagrangian duality between constrained estimation and control," Automatica, vol. 41, no. 6, pp. 935-944, 2005.

[6] R. E. Mortensen, "Maximum-likelihood recursive nonlinear filtering," Journal of Optimization Theory and Applications, vol. 2, no. 6, pp. 386-394, 1968.

[7] W. Fleming and S. Mitter, "Optimal control and nonlinear filtering for nondegenerate diffusion processes," Stochastics, vol. 8, pp. 63-77, 1982.

[8] S. K. Mitter and N. J. Newton, "A variational approach to nonlinear estimation," SIAM journal on control and optimization, vol. 42 , no. 5 , pp. 1813-1833, 2003.

[9] H. Ruiz and H. J. Kappen, "Particle smoothing for hidden diffusion processes: Adaptive path integral smoother," IEEE Transactions on Signal Processing, vol. 65, no. 12, pp. 3191-3203, 2017.

[10] R. Chetrite and H. Touchette, "Variational and optimal control representations of conditioned and driven processes," Journal of Statistical Mechanics: Theory and Experiment, vol. 2015, no. 12, p. P12001, 2015.
[11] A. Taghvaei, J. de Wiljes, P. G. Mehta, and S. Reich, "Kalman filter and its modern extensions for the continuous-time nonlinear filtering problem," Journal of Dynamic Systems, Measurement, and Control, vol. 140, no. 3, p. 030904, 2018

[12] R. E. Kalman and R. S. Bucy, "New results in linear filtering and prediction theory," Journal of basic engineering, vol. 83, no. 1, pp. 95-108, 1961.

[13] T. Yang, P. G. Mehta, and S. P. Meyn, "Feedback particle filter," IEEE Transactions on Automatic Control, vol. 58, no. 10, pp. 2465-2480, October 2013.

[14] T. Yang, R. S. Laugesen, P. G. Mehta, and S. P. Meyn, "Multivariable feedback particle filter," Automatica, vol. 71, pp. 10-23, 2016.

[15] A. Taghvaei and P. G. Mehta, "An optimal transport formulation of the linear feedback particle filter," in American Control Conference (ACC), 2016. IEEE, 2016, pp. 3614-3619.

[16] P. Billingsley, Probability and measure, ser. Wiley series in probability and mathematical statistics. Wiley, 1986.

[17] K. Bergemann and S. Reich, "An ensemble kalman-bucy filter for continuous data assimilation," Meteorologische Zeitschrift, vol. 21, no. 3, pp. 213-219, 2012.

\section{APPENDIX}

\section{A. Proof of Theorem 1}

Express the filter (9) as

$$
\bar{S}_{T}=c_{T}^{\top}\left(\bar{X}_{0}-m_{0}\right)+M_{T}\left(b_{T}, u_{t}\right)
$$

where

$$
M_{T}\left(b_{T}, u_{t}\right):=b_{T}^{\top} m_{0}+\int_{0}^{T} u_{t} \mathrm{~d} Z_{t}=\mathrm{E}\left[\bar{S}_{T} \mid \mathcal{Z}_{T}\right]
$$

is the conditional mean of $\bar{S}_{T}$.

The first objective involves minimization of the meansquared error (filter variance):

$$
\mathrm{E}\left[\left|\mathrm{E}\left[\bar{S}_{T} \mid \mathcal{Z}_{T}\right]-a^{\top} X_{T}\right|^{2}\right]=\underbrace{\mathrm{E}\left[\left|M_{T}-a^{\top} X_{T}\right|^{2}\right]}_{\text {term (i) }}
$$

The second objective function in (10) is expressed as a sum of two terms:

$$
\begin{gathered}
\mathrm{E}\left[\left|\mathrm{E}\left[\bar{S}_{T}^{2} \mid \mathcal{Z}_{T}\right]-\left(a^{\top} X_{T}\right)^{2}\right|^{2}\right]=\mathrm{E}\left[\left|c_{T}^{\top} \Sigma_{0} c_{T}+M_{T}^{2}-\left(a^{\top} X_{T}\right)^{2}\right|^{2}\right] \\
=\underbrace{\operatorname{var}\left[\left(a^{\top} X_{T}\right)^{2}-M_{T}^{2}\right]}_{\text {term (ii) }}+\underbrace{\left(c_{T}^{\top} \Sigma_{0} c_{T}-\mathrm{E}\left[\left(a^{\top} X_{T}\right)^{2}-M_{T}^{2}\right]\right)^{2}}_{\text {term (iii) }}
\end{gathered}
$$

The optimization of the three terms (i)-(iii) is the subject of the three steps in this proof.

1) In step 1, term (i) is minimized by choosing the vector $b_{T}$ and the control $u_{t}$. The solution of this problem is given by classical duality.

2) In step 2, it is shown that solution thus obtained also minimizes term (ii).

3) In step 3, the vector $c_{T}$ is chosen to minimize the square term (iii). Its minimum value is 0 .

The details of the three steps appear next.

Step 1: The solution to the term (i) minimization problem is given by classic duality [1]. A dual process is introduced:

$$
\mathrm{d} y_{t}=-A_{t}^{\top} y_{t} \mathrm{~d} t+C_{t}^{\top} u_{t} \mathrm{~d} t, \quad y_{T}=a
$$

Since $\mathrm{d}\left(y_{t}^{\top} X_{t}\right)=u_{t}^{\top} C_{t} X_{t} \mathrm{~d} t+y_{t}^{\top} \mathrm{d} B_{t}$,

$$
a^{\top} X_{T}=y_{0}^{\top} X_{0}+\int_{0}^{T} u_{t}^{\top} C_{t} X_{t} \mathrm{~d} t+\int_{0}^{T} y_{t}^{\top} \mathrm{d} B_{t}
$$


Using (19) and $\mathrm{d} Z_{t}=C_{t} X_{t} \mathrm{~d} t+\mathrm{d} W_{t}$, one obtains

$$
M_{T}-a^{\top} X_{T}=b_{T}^{\top} m_{0}-y_{0}^{\top} X_{0}+\int_{0}^{T} u_{t}^{\top} \mathrm{d} W_{t}-\int_{0}^{T} y_{t}^{\top} \mathrm{d} B_{t}
$$

Squaring and taking expectations, the term (i) is expressed as

$$
\left(\left(b_{T}^{\top}-y_{0}^{\top}\right) m_{0}\right)^{2}+y_{0}^{\top} \Sigma_{0} y_{0}+\int_{0}^{T} y_{t}^{\top} Q_{t} y_{t}+u_{t}^{\top} R_{t} u_{t} \mathrm{~d} t
$$

With the minimizing choice of $b_{T}=y_{0}=: b_{T}^{*}$, the term (i) minimization problem is transformed into a linear quadratic (LQ) optimal control problem

$$
\min _{u_{t}} y_{0}^{\top} \Sigma_{0} y_{0}+\int_{0}^{T} y_{t}^{\top} Q_{t} y_{t}+u_{t}^{\top} R_{t} u_{t} \mathrm{~d} t
$$

subject to the dynamic constraints given by the dual system (21). form

The optimal control law is easily obtained in the feedback

$$
u_{t}=\mathrm{K}_{t}^{\top} y_{t}
$$

where the gain $\mathrm{K}_{t}:=\Sigma_{t} C^{\top} R_{t}^{-1}$ is obtained by solving the DRE (2b). Upon using the defintion (12) of the state transition matrix $\Phi(t, \tau)$,

$$
u_{t}=\mathrm{K}_{t}^{\top} y_{t}=\Phi(t ; T) y_{T}=\Phi(t ; T) a=: u_{T}^{*}
$$

We use the notation $\hat{S}_{T}$ to denote $M_{T}$ with the optimal choice of the parameters $b_{T}^{*}$ and $u_{t}^{*}$. Explicitly,

$$
\hat{S}_{T}:=M_{T}\left(b_{T}^{*}, u_{t}^{*}\right)=a^{\top} \Phi^{\top}(0 ; T) m_{0}+\int_{0}^{T} a^{\top} \Phi^{\top}(t ; T) \mathrm{K}_{t} \mathrm{~d} Z_{t}
$$

$\hat{S}_{T}$ has the following properties which are useful in the remainder of the proof:

1) $\hat{S}_{T}$ is the conditional mean, i.e., $\hat{S}_{T}=E\left[a^{\top} X_{T} \mid \mathcal{Z}_{T}\right]$.

2) The error $\left(a^{\top} X_{T}-\hat{S}_{T}\right)$ satisfies the orthogonal property whereby $\mathrm{E}\left[\left(a^{\top} X_{T}-\hat{S}_{T}\right) \hat{S}_{T}\right]=0$.

3) The optimal value $\mathrm{E}\left[\left|\hat{S}_{T}-a^{\top} X_{T}\right|^{2}\right]=a^{\top} \Sigma_{T} a$.

Step 2: The optimal parameters, $b_{T}^{*}$ and $u_{t}^{*}$, obtained in step 1 also minimize the term (ii). The reasoning is as follows: Note that the term (ii) involves minimization of the variance again by choosing $b_{T}$ and $u_{t}$. Since the resulting random variable $M_{T}^{2}$ is $\mathcal{Z}_{T}$-measurable, any minimizer of term (ii) is of the general form

$$
M_{T}^{2}=\mathrm{E}\left[\left(a^{\top} X_{T}\right)^{2} \mid \mathcal{Z}_{T}\right]+(\text { const. })
$$

Now, the conditional variance of $a^{\top} X_{T}$ is given by

$$
\begin{aligned}
\operatorname{var}\left[a^{\top} X_{T} \mid \mathcal{Z}_{T}\right] & =\mathrm{E}\left[\left(a^{\top} X_{T}-\mathrm{E}\left[a^{\top} X_{T} \mid \mathcal{Z}_{T}\right]\right)^{2} \mid \mathcal{Z}_{T}\right] \\
& =\mathrm{E}\left[\left(a^{\top} X_{T}-\mathrm{E}\left[a^{\top} X_{T} \mid \mathcal{Z}_{T}\right]\right)^{2}\right] \\
& =\mathrm{E}\left[\left(a^{\top} X_{T}-\hat{S}_{T}\right)^{2}\right]=a^{\top} \Sigma_{T} a
\end{aligned}
$$

The second equality comes from the fact that orthogonality implies independence for a Gaussian random variable. Since

$$
\begin{aligned}
\operatorname{var}\left[a^{\top} X_{T} \mid \mathcal{Z}_{T}\right] & =\mathrm{E}\left[\left(a^{\top} X_{T}\right)^{2} \mid \mathcal{Z}_{T}\right]-\left(\mathrm{E}\left[a^{\top} X_{T} \mid \mathcal{Z}_{T}\right]\right)^{2} \\
& =\mathrm{E}\left[\left(a^{\top} X_{T}\right)^{2} \mid \mathcal{Z}_{T}\right]-\hat{S}_{T}^{2}
\end{aligned}
$$

we have

$$
\hat{S}_{T}^{2}=\mathrm{E}\left[\left(a^{\top} X_{T}\right)^{2} \mid \mathcal{Z}_{T}\right]-a^{\top} \Sigma_{T} a
$$

Comparing with (23), it follows that $M_{T}=\hat{S}_{T}$ is a minimizer of the term (ii). Consequently, $b_{T}^{*}$ and $u_{t}^{*}$ are optimal choices for minimizing term (ii) as well. It is noted that $\hat{S}_{T}$ (defined in (22)) is the only solution that simultaneously minimizes both terms (i) and (ii).

Step 3: Now that the vector $b_{T}$ and the control $u_{t}$ have been obtained, the vector $c_{T}$ is chosen to make the square term (iii) zero. We have

$$
\begin{aligned}
c_{T}^{\top} \Sigma_{0} c_{T} & =\mathrm{E}\left[\left(a^{\top} X_{T}\right)^{2}-\left(\hat{S}_{T}\right)^{2}\right] \\
& =\mathrm{E}\left[\left(a^{\top} X_{T}\right)^{2}-\left(\hat{S}_{T}\right)^{2}\right]-2 \mathrm{E}\left[\left(a^{\top} X_{T}-\hat{S}_{T}\right) \hat{S}_{T}\right] \\
& =\mathrm{E}\left[\left|a^{\top} X_{T}-\hat{S}_{T}\right|^{2}\right]=a^{\top} \Sigma_{T} a
\end{aligned}
$$

Therefore, the minimizing choice of $c_{T}$ is obtained by solving the scalar equation

$$
c_{T}^{\top} \Sigma_{0} c_{T}=a^{\top} \Sigma_{T} a
$$

It is straightforward to verify that $c_{T}=\Psi(0 ; T) a$ is a solution of this equation. A more constructive proof follows from introducing a backward-time process

$$
\frac{\mathrm{d} \xi_{t}}{\mathrm{~d} t}=\left(-A_{t}^{\top}+\frac{1}{2} C_{t}^{\top} \mathrm{K}_{t}^{\top}-\frac{1}{2} \Sigma_{t}^{-1} Q_{t}\right) \xi_{t}, \quad \xi_{T}=a
$$

Since $\Sigma_{t}$ is a solution of the DRE (2b), it is easy to then verify that $\mathrm{d}\left(\xi_{t}^{\top} \Sigma_{t} \xi_{t}\right)=0$, and so $\xi_{0}^{\top} \Sigma_{0} \xi_{0}=a^{\top} \Sigma_{T} a$. Thus, $c_{T}=\xi_{0}=\Psi(0 ; T) a$ is a solution.

The proof for exactness is deferred to the following Sections.

\section{B. Proof of Proposition 2}

The equation (15) for the optimal estimator has the following form: $\bar{S}_{T}=a^{\top} \bar{X}_{T}$. Upon denoting the (arbitrary) final time $T$ simply as $t$, one writes

$$
\bar{X}_{t}=\Psi^{\top}(0 ; t)\left(\bar{X}_{0}-m_{0}\right)+\bar{m}_{t}
$$

where $\bar{m}_{t}=\Phi^{\top}(0 ; t) m_{0}+\int_{0}^{t} \Phi^{\top}(s ; t) \mathrm{K}_{s} \mathrm{~d} Z_{s}=\mathrm{E}\left[\bar{X}_{t} \mid \mathcal{Z}_{t}\right]$.

Upon differentiating (24), and using the formulae (12) and (13) for the state transition matrices,

$$
\begin{aligned}
\mathrm{d} \bar{X}_{t}= & \left(A_{t}-\mathrm{K}_{t} C_{t}\right) \Phi^{\top}(0 ; t) m_{0} \mathrm{~d} t \\
& +\left(A_{t}-\frac{1}{2} \mathrm{~K}_{t} C_{t}+\frac{1}{2} Q_{t} \Sigma_{t}^{-1}\right) \Psi^{\top}(0 ; t)\left(\bar{X}_{0}-m_{0}\right) \mathrm{d} t \\
& +\left(\int_{0}^{t}\left(A_{t}-\mathrm{K}_{t} C_{t}\right) \Phi^{\top}(s ; t) \mathrm{K}_{s} \mathrm{~d} Z_{s}\right) \mathrm{d} t+\mathrm{K}_{t} \mathrm{~d} Z_{t} \\
= & \left(A_{t}-\mathrm{K}_{t} C_{t}\right) \bar{X}_{t} \mathrm{~d} t+\left(\frac{1}{2} \mathrm{~K}_{t} C_{t}+\frac{1}{2} Q_{t} \Sigma_{t}^{-1}\right)\left(\bar{X}_{t}-\bar{m}_{t}\right) \mathrm{d} t
\end{aligned}
$$

This yields the recursive formula for the filter. The proof of exactness of this filter has already appeared in [15] (see also Prop. 1). It is also a special case of the more general stochastic filter whose exactness proof appears in the Sec. D. 


\section{Proof of Theorem 2 and Proposition 3}

Express the filter $(17 \mathrm{c})$ as

$$
\bar{S}_{T}=M_{T}+c_{T}^{\top}\left(\bar{X}_{0}-m_{0}\right)+\int_{0}^{T} v_{t}^{\top} \mathrm{d} \bar{B}_{t}+\int_{0}^{T} w_{t}^{\top} \mathrm{d} \bar{W}_{t}
$$

where $M_{T}=M_{T}\left(b_{T}, u_{t}\right)=\mathrm{E}\left[\bar{S}_{T} \mid \mathcal{Z}_{T}\right]$ is as defined in (19). The other three terms model the effects of randomness due to the initial condition, process noise and the measurement noise, respectively.

Since both the noise terms have zero-mean, these do not affect the terms (i) and (ii) as introduced in the proof of Thm. 1. The counterpart of (20) now is:

$$
\begin{aligned}
& \mathrm{E}\left[\left|\mathrm{E}\left[\bar{S}_{T}^{2} \mid \mathcal{Z}_{T}\right]-\left(a^{\top} X_{T}\right)^{2}\right|^{2}\right]=\underbrace{\operatorname{var}\left[\left(a^{\top} X_{T}\right)^{2}-M_{T}^{2}\right]}_{\text {term (ii) }} \\
& +\underbrace{\left(c_{T}^{\top} \Sigma_{0} c_{T}+\int_{0}^{T} v_{t}^{\top} Q_{t} v_{t}+w_{t}^{\top} R_{t} w_{t} \mathrm{~d} t-\mathrm{E}\left[\left(a^{\top} X_{T}\right)^{2}-M_{T}^{2}\right]\right)^{2}}_{\text {term (iii) }}
\end{aligned}
$$

Since the terms (i) and (ii) are identical to the ones in the proof the Thm. 1, the steps 1 and 2 apply in an identical manner. The optimal solution for $b_{T}$ and $u_{t}$ is thus the same as before. The optimal $M_{T}$ is given by $\hat{S}_{T}$ (see (22)).

Upon setting the square term (iii) ${ }^{\prime}$ to its minimum value, zero, the scalar equation for $c_{T}$ is now given by

$$
c_{T}^{\top} \Sigma_{0} c_{T}=a^{\top} \Sigma_{T} a-\int_{0}^{T} v_{t}^{\top} Q_{t} v_{t}+w_{t}^{\top} R_{t} w_{t} \mathrm{~d} t
$$

This equation has many solutions. We pick one solution by introducing the following modification of the process $\xi_{t}$ :

$$
\frac{\mathrm{d} \xi_{t}}{\mathrm{~d} t}=\left(-A_{t}^{\top}+\frac{1+\gamma_{2}^{2}}{2} C_{t}^{\top} \mathrm{K}_{t}^{\top}-\frac{1-\gamma_{1}^{2}}{2} \Sigma_{t}^{-1} Q_{t}\right) \xi_{t}, \xi_{T}=a
$$

where $\gamma_{1}$ and $\gamma_{2}$ are arbitrary constants. Then

$$
\mathrm{d}\left(\xi_{t}^{\top} \Sigma_{t} \xi_{t}\right)=\gamma_{1}^{2} \xi_{t}^{\top} Q_{t} \xi_{t} \mathrm{~d} t+\gamma_{2}^{2} \xi_{t}^{\top} \mathrm{K}_{t} R_{t} \mathrm{~K}_{t}^{\top} \xi_{t} \mathrm{~d} t, \quad \xi_{T}=a
$$

Therefore, upon setting $v_{t}=\gamma_{1} \xi_{t}$ and $w_{t}=\gamma_{2} \mathrm{~K}_{t}^{\top} \xi_{t}$, one obtains

$$
\xi_{0}^{\top} \Sigma_{0} \xi_{0}=a^{\top} \Sigma_{T} a-\int_{0}^{T} v_{t}^{\top} Q_{t} v_{t}+w_{t}^{\top} R_{t} w_{t} \mathrm{~d} t
$$

and thus

$$
\begin{aligned}
c_{T} & =\xi_{0}=\Psi(0 ; T) a \\
v_{t} & =\gamma_{1} \Psi(t ; T) a \\
w_{t} & =\gamma_{2} \mathrm{~K}_{t}^{\top} \Psi(t ; T) a
\end{aligned}
$$

solves (25).

Using these parameters, the estimator is $\bar{S}_{T}=a^{\top} \bar{X}_{T}$ where

$$
\begin{aligned}
\bar{X}_{t}= & \Phi^{\top}(0 ; t) m_{0}+\Psi^{\top}(0 ; t)\left(\bar{X}_{0}-m_{0}\right) \\
& +\int_{0}^{t} \Phi^{\top}(s ; t) \mathrm{K}_{s} \mathrm{~d} Z_{s}+\gamma_{1} \int_{0}^{t} \Psi^{\top}(s ; t) \mathrm{d} \bar{B}_{s} \\
& +\gamma_{2} \int_{0}^{t} \Psi^{\top}(s ; t) \mathrm{K}_{s} \mathrm{~d} \bar{W}_{s}
\end{aligned}
$$

Its differential form is easily obtained as

$$
\begin{aligned}
\mathrm{d} \bar{X}_{t}= & A_{t} \bar{X}_{t} \mathrm{~d} t+\gamma_{1} \mathrm{~d} \bar{B}_{t}+\frac{1-\gamma_{1}^{2}}{2} Q_{t} \Sigma_{t}^{-1}\left(\bar{X}_{t}-\bar{m}_{t}\right) \mathrm{d} t \\
& +\mathrm{K}_{t}\left(\mathrm{~d} Z_{t}-C_{t}\left(\frac{\left(1+\gamma_{2}^{2}\right) \bar{X}_{t}+\left(1-\gamma_{2}^{2}\right) \bar{m}_{t}}{2}\right) \mathrm{d} t+\gamma_{2} \mathrm{~d} \bar{W}_{t}\right)
\end{aligned}
$$

\section{Proof of the exactness of the filter}

The filter (26) is a linear sde, the initial condition $\bar{X}_{0}$ is Gaussian, and the noise terms are also Gaussian. Therefore, the solution $\bar{X}_{t}$ is Gaussian for all $t>0$. Thus, in order to show exactness, all we need to show is that the equations for the conditional mean and the variance evolve according to the Kalman filter equations.

Upon taking a conditional expectation of (26),

$$
\mathrm{d} \bar{m}_{t}=A_{t} \bar{m}_{t}+\mathrm{K}_{t}\left(\mathrm{~d} Z_{t}-C_{t} \bar{m}_{t} \mathrm{~d} t\right), \quad \bar{m}_{0}=m_{0}
$$

This is the same as the equation (2a) of the Kalman filter.

Next define the error process $e_{t}:=\bar{X}_{t}-\bar{m}_{t}$. The equation for the error process is given by

$$
\begin{aligned}
\mathrm{d} e_{t}= & A_{t} e_{t} \mathrm{~d} t+\frac{1-\gamma_{1}^{2}}{2} Q_{t} \Sigma_{t}^{-1} e_{t} \mathrm{~d} t \\
& -\mathrm{K}_{t} C_{t} \frac{1+\gamma_{2}^{2}}{2} e_{t} \mathrm{~d} t+\gamma_{1} \mathrm{~d} \bar{B}_{t}+\gamma_{2} \mathrm{~K}_{t} \mathrm{~d} \bar{W}_{t}
\end{aligned}
$$

Upon squaring the taking expectations, one obtains the differential equation for the variance $\bar{\Sigma}_{t}=\operatorname{var}\left(\bar{X}_{t}\right)$ as follows:

$$
\begin{aligned}
\frac{\mathrm{d} \bar{\Sigma}_{t}}{\mathrm{~d} t}= & A_{t} \bar{\Sigma}_{t}+\bar{\Sigma}_{t} A_{t}^{\top}+\frac{1-\gamma_{1}^{2}}{2}\left(Q_{t} \Sigma_{t}^{-1} \bar{\Sigma}_{t}+\bar{\Sigma}_{t} \Sigma_{t}^{-1} Q_{t}\right) \\
& -\frac{1+\gamma_{2}^{2}}{2}\left(\mathrm{~K}_{t} C_{t} \bar{\Sigma}_{t}-\bar{\Sigma}_{t} C^{\top} \mathrm{K}_{t}^{\top}\right)+\gamma_{1}^{2} Q_{t}+\gamma_{2}^{2} \mathrm{~K}_{t} R_{t} \mathrm{~K}_{t}^{\top}
\end{aligned}
$$

with initial condition $\bar{\Sigma}_{0}=\Sigma_{0}$. This is a linear ode (in $\bar{\Sigma}_{t}$ ). It thus admits a unique solution. It is straightforward to verify that $\bar{\Sigma}_{t}=\Sigma_{t}$ in fact solves the equation (recall that $\Sigma_{t}$ is the solution to the DRE (2b) with initial condition $\Sigma_{0}$ ).

Therefore, the filter (26) is exact for all time $t \geq 0$. 